\title{
Fractal Dimension of the EEG for Detection of Behavioural Microsleeps
}

\author{
M. T. R. Peiris ${ }^{1,2,3}$, R. D. Jones ${ }^{1,2,3,4}$, P. R. Davidson ${ }^{1,3}$, P. J. Bones ${ }^{1,2}$, D. J. Myall ${ }^{1,4}$ \\ ${ }^{1}$ Van der Veer Institute for Parkinson's and Brain Research, Christchurch, New Zealand \\ ${ }^{2}$ Electrical and Computer Engineering, University of Canterbury, Christchurch, New Zealand \\ ${ }^{3}$ Medical Physics and Bioengineering, Christchurch Hospital, Christchurch, New Zealand \\ ${ }^{4}$ Medicine, Christchurch School of Medical and Health Sciences, University of Otago, Christchurch, New Zealand
}

\begin{abstract}
The fractal dimension (FD) of EEG has been shown to be of value in the detection of epileptic seizures. In this paper, we assess its usefulness in detecting behavioural microsleeps. Fifteen non-sleep-deprived normal subjects performed two 1-hour sessions of a continuous tracking task while EEG, EOG and facial video were recorded. Higuchi's algorithm was used to calculate the FD of the EEG. Video lapses were scored independently from tracking performance by a human rater. A subset of data was rated independently by three human raters observing both tracking performance and the video rating to identify behavioural microsleep events. The mean point-biserial correlation between FD and the mean human rating was $\mathbf{- 0 . 2 1 3}$ indicating modest agreement. Crossvalidated detection performance of the FD was poor with a mean correlation $(\varphi=\mathbf{- 0 . 0 9 9})$. This suggests that, on its own, FD of the EEG is unlikely to be useful for detecting microsleeps.
\end{abstract}

Keywords-Alertness, behavioural microsleeps, fractal dimension, visuomotor tracking

\section{INTRODUCTION}

Microsleeps are brief periods of sleep during which there is a marked reduction in behavioural responsiveness [1]. They have been shown to occur frequently when subjects are tired [2]. Other than drowsiness, there is often no advance warning of sleep/microsleep onset. In several studies, microsleeps are defined as short bursts of sleep in the EEG/EOG [1, 3-6]. While associated with drowsiness, EEG signatures of sleep in the transitional phases (stages 1 and 2) are not well correlated with behavioural sleep [7]. We prefer to consider microsleep a behavioural phenomenon and emphasize that our usage is distinct from an EEG-defined microsleep. We refer to behavioural microsleeps as lapses.

Lapses are of particular concern for occupational groups with a need to maintain a high level of alertness for extended periods, such as truck drivers, locomotive drivers, pilots, air traffic controllers, and surgeons [8, 9]. Diminished alertness and responsiveness in these occupations can have disastrous consequences, including

This research was financially supported by Foundation for Research Science and Technology and the University of Canterbury, Christchurch, New Zealand. multiple fatalities. Microsleeps are also of considerable concern to the general population when driving.

FD of the EEG has previously been found useful in characterizing EEG changes in bipolar disorder [11] and during epileptic seizures [12]. FD has also been referred to as the Hausdorff dimension, Hausdorff-Besicovitch dimension or the capacity dimension. FD measures the complexity of a signal [10], and varies between 1.0 (low complexity) and 2.0 (high complexity).

In a previous study we explored the characteristics of lapsing by recording full-head EEG, eye movements, and video from 15 subjects undertaking a 1-D continuous tracking task over two $1 \mathrm{hr}$ sessions [13]. In this paper, we investigate the relationship between the Higuchi FD of the EEG and behavioural microsleeps using data from our earlier study.

\section{Methodology}

\section{A. Tracking Study}

Data were recorded from 15 normal healthy male volunteers aged $18-36$ years $($ mean $=26.5)$ while they performed a tracking task. None had a history of neurological or sleep disorders and all had visual acuities of $6 / 9(=20 / 30)$ or better in each eye.

The tracking task was generated by the sensory-motor tests program SMTests ${ }^{\mathrm{TM}}$ which displayed a continuous target signal on a computer screen [14]. Eye-to-screen distance was $136 \mathrm{~cm}$. Subjects used a steering wheel $(39.5$ $\mathrm{cm}$ diameter; wheel-to-screen gain $=1.075 \mathrm{~mm} / \mathrm{deg}$ ) to control an arrow-shaped cursor, located at the bottom of the screen. Their task was to keep the arrowhead as close as possible to a pseudo-random target (bandwidth $0.164 \mathrm{~Hz}$, period $128 \mathrm{~s}$ ) which scrolled down the screen at $21.8 \mathrm{~mm} / \mathrm{s}$ (providing 8-s preview [15]). The angular position of the wheel was sampled at $64 \mathrm{~Hz}$ and a video camera was used to record head and facial features of a subject during the session ( $25 \mathrm{~Hz}$ frame rate).

EEG was recorded from electrodes at 16 scalp locations and digitized at $256 \mathrm{~Hz}$ with a 16 bit A-D converter. The following bipolar derivations were used in the analysis: Fp1-F7, F7-T3, T3-T5, T5-O1, Fp2-F8, F8-T4, T4-T6, T6-O2, Fp1-F3, F3-C3, C3-P3, P3-O1, Fp2-F4, F4-C4, C4-P4, P4-O2. 
Subjects attended two sessions held on different days (separated by a mean of 17 days, range 7-50) and refrained from consumption of stimulants/depressants for 4 hours prior to each experimental session. All sessions were held between 12.30 p.m. and 5.00 p.m. Subjects were instructed to perform the tracking task continuously for one hour and, aside from eye blinks, to keep their eyes open as much as possible during the task.

\section{B. Analysis}

Video rating: The video recording of each session was rated by an expert, without knowledge of the corresponding tracking performance, on a 7 level scale: alert, distracted, forced eye closure while alert, light drowsy, deep drowsy, lapse, and sleep. The rating was based on eye movements and other behavioural markers such as loss of facial tone, slow eyelid closure, yawning and nodding. The timeresolution of the video rating was $1 \mathrm{~s}$. The rater was 'blind' to the tracking performance of subjects to ensure independence.

Expert rating of lapses: The video rating by itself does not make optimal use of the available behavioural data. Ideally, a metric with good temporal resolution indicating overall lapse likelihood given all available behavioural data is desirable. Since the opinions of experts vary regarding the onset of lapses, a short study was conducted to establish the mean opinion of human experts on the overall probability of a lapse given both the tracking and video data.

Three human experts rated 12 min of data from a randomly-chosen session from each subject. The 12 minutes comprised the first 2 min (baseline) of each session followed by the epoch between 30 and $40 \mathrm{~min}$ from the start of the session. Raters were required to mark transitions between the levels of a 5-point scale capturing the degree of certainty that a lapse was occurring: Definitely Not Lapse, Probably Not Lapse, Unsure, Probable Lapse, and Definite Lapse. Ratings were based upon tracking behaviour and the human video rating displayed concurrently on a computer screen.

The mean of the three ratings was used to determine when a lapse occurred. A mean rating $>3$ was considered a lapse. We refer to this as the Lapse Index (LI). The baseline data of the mean rating was not used in the analysis of the FD.

Fractal dimension: We used Higuchi's algorithm [16] to estimate the FD because it is computationally efficient and provides a stable estimate of FD using a lower number of samples of data $(\mathrm{N} \geq 125)$ than other FD algorithms [10], which allowed us to estimate FD with good temporal resolution.

The Higuchi algorithm generates multiple time series from a given EEG segment consisting of $N$ samples $(x(i), i=$ $1, \ldots, N)$. Starting from the $m^{\text {th }}$ data point of the signal, points are sampled with scale size $k$ such that successive samples are $k$ data points apart. This process is repeated for different starting points $(m=1, \ldots, k)$ and different scale sizes $\left(1, \ldots, k_{\max }\right)$ such that, for each scale size $k$, a set of time series is obtained:

$$
x_{m}^{k}=\{x(m), x(m+k), \ldots, x(m+\lfloor(N-m) / k\rfloor) k\}
$$

for $m=1,2, \ldots, k$, where $\lfloor a\rfloor$ denotes the integer part of $a$.

For each time series $x_{m}^{k}$, the absolute differences between each two successive data points are summed to calculate the vertical length of the signal measured with the scale size $k$, starting at the $m^{\text {th }}$ data point:

$$
L_{m}(k)=\frac{\sum_{i=1}^{\lfloor(N-m) / k\rfloor}|x(m+i k)-x(m+(i-1) k)|(n-1)}{\lfloor(N-m) / k\rfloor k} .
$$

The length of the EEG segment $L(k)$ is the mean of $L_{m}(k)$ values, for $m=1, \ldots, k$. This procedure is repeated for each $k$ ranging from 1 to $k_{\max }$ yielding a sum of average lengths $L(k)$ for each $k$ :

$$
L(k)=\sum_{m=1}^{k} L_{m}(k) .
$$

If the value of $L(k)$ is proportional to $k^{-d}$, then the signal would be fractal-like and have the dimension $d$. The angular coefficient of the linear regression of the graph $\log (L(k)) v s$. $\log (1 / k)$ gives an estimate of the fractal dimension $d$.

We used parameters suggested by Accardo et al. [10] for estimating the FD of the EEG $\left(k_{\max }=6\right.$, window size, $N$ $=256$ samples $(1 \mathrm{~s})$, window overlap $=75 \%)$. We preprocessed the EEG using a notch filter to remove $50 \mathrm{~Hz}$ interference, independent components analysis to remove eye blink artifacts $[17,18]$, and a low-pass filter to remove noise above $70 \mathrm{~Hz}$ as these artifacts have been shown to affect the FD [10]. These parameters allowed a temporal resolution of $1 \mathrm{~Hz}$ for the FD. Before comparison with LI the FD was low-pass filtered (cutoff $f=0.125 \mathrm{~Hz}$ ). The cutoff frequency was determined by finding the strongest point-biserial correlation [19] between FD and LI over a range of cut-off values.

Performance evaluation: Subjects who lapsed at least once during the $10 \mathrm{~min}$ of rating were selected for analysis $(\mathrm{N}=8)$. An example of the LI, FD and EEG for the P4-O2 derivation is shown in Fig 1.

For lapse detection we wanted to derive a binary (lapse/no lapse) decision from the FD. This required applying a binary decision threshold to the continuous FD index. We used the phi coefficient $\varphi$ (Pearson correlation coefficient between two binary variables [19]), to measure the agreement between the resulting binary FD and LI. Stratified four-fold cross-validation was used to evaluate the performance of the FD lapse detector. The 8 subjects were divided into 4 sets of 2 . A single optimal threshold was determined from the three sets by finding the level that yielded the strongest correlation, and this level was applied to the remaining set. The same process was repeated 3 
times so that each pair of subjects was used as the test set once. The whole procedure was repeated 10 times with different random subject orders. The mean correlations across repetitions are reported.

Since optimum threshold levels may differ between subjects, we also tested whether performance improved if a unique level was set for each individual. The first $5 \mathrm{~min}$ of data from the subject was used to determine the optimal FD threshold for a particular EEG channel and the second $5 \mathrm{~min}$ used to calculate $\varphi$. This procedure was performed on each EEG channel of each subject.

Cross-correlation analysis was also performed to determine if there was a lead/lag between FD and LI. For each subject, the delay at which the peak cross-correlation occurred in all 16 derivations was measured. The derivations were divided into 4 groups (pre-frontal-frontal, frontal-central, central-parietal, parietal-occipital). The $t$ test was used to determine if the peak correlation occurred at a significant lead/lag for any of the groups.
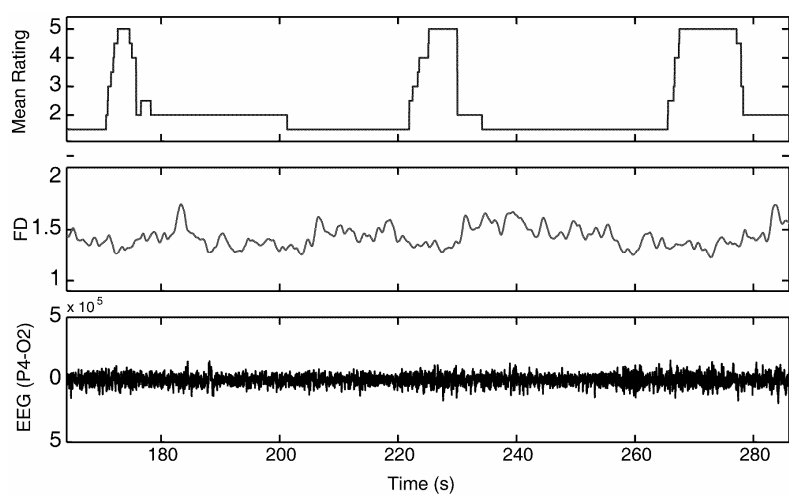

Fig. 1. A 2 min sample segment of the mean rating, Higuchi FD, and EEG (P4-O2). The Lapse Index (LI) was derived from the mean rating by placing a threshold at 3 .

\section{RESULtS}

\section{A. Correlation with Lapse Index}

Fig. 2 shows the mean point-biserial correlations between the LI and continuous FD for each of the 16 EEG derivations. The overall mean point-biserial correlation across all derivations was -0.213 (range: $-0.155--0.261$ ) with the highest value in $\mathrm{P} 4-\mathrm{O} 2$.

Cross-correlation analysis revealed that the FD leads the LI (mean lead $=1.47 \mathrm{~s}$ ) across the parietal-occipital derivations $(t$-test; $p=0.012)$. The corresponding increase in absolute mean correlation at this lead was minimal (mean $\varphi$ $=0.02$ ), so a lag of zero was used for the remaining analysis.

\section{B. Lapse Detection}

In our first attempt at using FD to detect lapses, we determined a single optimal decision threshold for each channel, to be applied to the FD from all subjects. We used 4-fold cross validation to assess the performance of this detector. Fig. 3 shows mean $\varphi$ for the 16 EEG derivations,

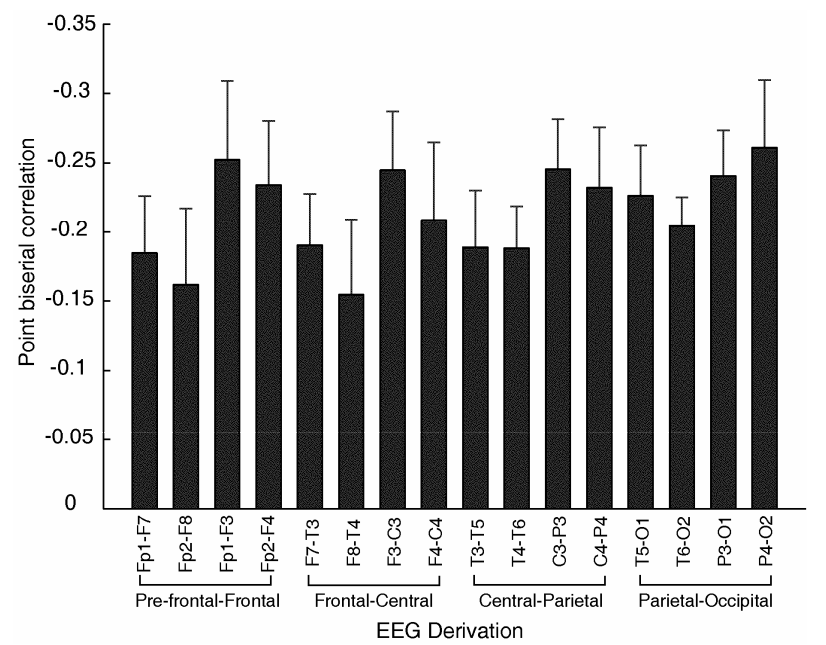

Fig. 2. Mean point-biserial correlation between the continuous FD and the binary lapse index over all EEG derivations $(\mathrm{N}=8)$ Standard error bars are also shown.

across 8 subjects. The overall mean $\varphi$ was -0.099 (range $=-$ $0.041--0.165)$ indicating poor performance.

Since the optimum threshold level may vary between subjects we looked at extent to which the mean $\varphi$ improved if a different optimum threshold was chosen for each

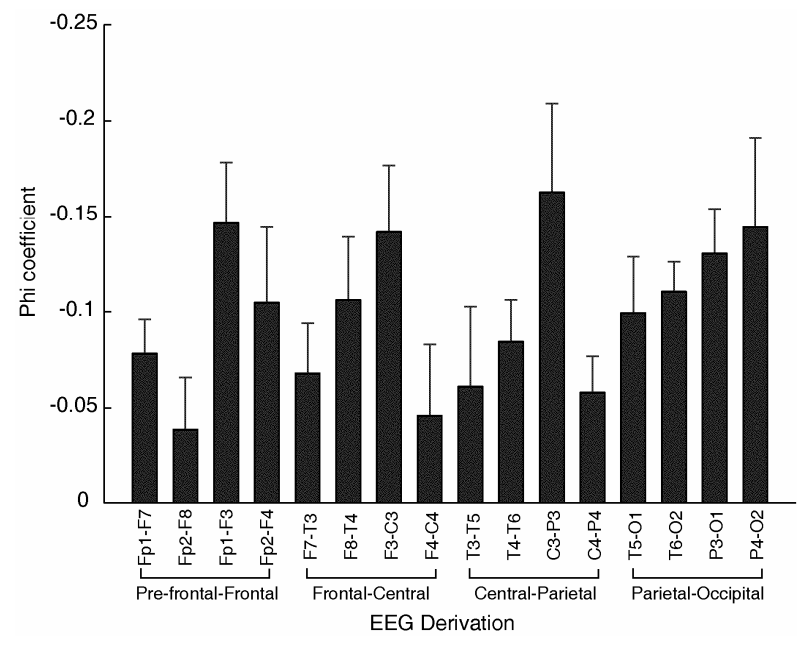

Fig. 3. Mean $\varphi$ coefficients between the FD and the Lapse Index over all EEG derivations $(\mathrm{N}=8)$ from 4-fold cross-validation analysis. Standard error bars are also shown.

subject. Results showed a slight improvement with mean $\varphi$ $=-0.128$ over 16 derivations (range $=-0.085--0.175$ ).

Repeating the analysis with noisy EEG data (i.e., contaminated with power line interference, eye blink artifacts) showed only a slight decrease in the overall mean point-biserial correlation from -0.213 to -0.188 . Single threshold detector performance also reduced slightly (mean $\varphi=-0.096)$. 


\section{DISCUSSION}

Our results show that the correlation between FD of the EEG and behavioural microsleeps during a visuomotor tracking task is modest. The correlation is negative, indicating that the EEG tends to become less complex during lapses.

Cross-validation analysis showed that a lapse detector would perform poorly. Optimum performance using a single threshold value applied to the FD of all subjects was on C3P3 $(\varphi=-0.167)$. Poor performance could conceivably be due to inter-subject variations in optimum threshold levels. Applying optimum individual thresholds to the FD of each subject improved detector performance slightly.

We found that the peak correlation occurred at a lead $(1.47 \mathrm{~s})$ in the parietal-occipital derivations. Theta activity, which has been shown to increase prior to auditory lapses [20], may account for this effect. No distinct spatial patterns were found in the correlation coefficients across derivations.

The modest improvement in mean correlation after artifact removal was surprising, especially since mains frequency interference has been shown to have a considerable effect [10]. However, its adverse effects may be minimal if the interference is relatively constant and when looking at relative rather than absolute variations in the FD.

The optimal LPF for FD was $0.125 \mathrm{~Hz}$, indicating that changes in the FD are relatively slow and that the observed correlation effect might be due to slow variations in alertness level rather than lapses.

\section{REFERENCES}

[1] Y. Harrison and J. A. Horne, "Occurrence of 'microsleeps' during daytime sleep onset in normal subjects," Electroencephalogr. Clin. Neurophysiol., vol. 98, pp. 411-416, 1996.

[2] S. K. L. Lal, A. Craig, P. Boord, L. Kirkup, and H. Nguyen, "Development of an algorithm for an EEG-based driver fatigue countermeasure," J. Safety Res., vol. 34, pp. 321-328, 2003.

[3] U. Hemmeter, R. Bischof, M. Hatzinger, E. Seifritz, and E. HolsboerTrachsler, "Microsleep during partial sleep deprivation in depression," Biol. Psychiat., vol. 43, pp. 829-839, 1998.

[4] B. Priest, C. Brichard, G. Aubert, G. Liistro, and D. O. Rodenstein, "Microsleep during a simplified maintenance of wakefulness test," $\mathrm{Am}$. J. Resp. Crit. Care, vol. 163, pp. 1619-1625, 2001.
[5] V. Valley and R. Broughton, "The physiological (EEG) nature of drowsiness and its relation to performance deficits in narcoleptics," Electroencephalogr. Clin. Neurophysiol., vol. 55, pp. 243-251, 1983.

[6] V. L. Tirunahari, S. A. Zaidi, R. Sharma, J. Skurnick, and H. Ashtyani, "Microsleep and sleepiness: a comparison of multiple sleep latency test and scoring of microsleep as a diagnostic test for excessive daytime sleepiness," Sleep Med., vol. 4, pp. 63-67, 2003.

[7] R. D. Ogilvie, "The process of falling asleep," Sleep Med. Rev., vol. 5, pp. 247-270, 2001.

[8] F. Sagberg, "Road accidents caused by drivers falling asleep," Accid. Anal. Prev., vol. 31, pp. 639-649, 1999.

[9] P. Cummings, T. D. Koepsell, J. M. Moffat, and F. P. Rivara, "Drowsiness, counter-measures to drowsiness, and the risk of a motor vehicle crash," Inj. Prev., vol. 7, pp. 194-9, 2001.

[10] A. Accardo, M. Affinito, M. Carrozzi, and F. Bouquet, "Use of the fractal dimension for the analysis of electroencephalographic time series," Biol. Cybern., vol. 77, pp. 339-50, 1997.

[11] B. Bahrami, R. Seyedsadjadi, B. Babadi, and M. Noroozian, "Brain complexity increases in mania," Neuroreport, vol. 16, pp. 187-91, 2005 .

[12] E. T. Bullmore, M. J. Brammer, P. Bourlon, G. Alarcon, C. E. Polkey, R. Elwes, and C. D. Binnie, "Fractal analysis of electroencephalographic signals intracerebrally recorded during 35 epileptic seizures: evaluation of a new method for synoptic visualisation of ictal events," Electroencephalogr. Clin. Neurophysiol., vol. 91, pp. 337-345, 1994.

[13] M. T. R. Peiris, R. D. Jones, G. Carroll, J., and P. J. Bones, "Investigation of lapses of consciousness using a tracking task: preliminary results," Proc. 26th Int. Conf. IEEE Eng. Med. Biol. Soc., pp. 4721-4724, 2004.

[14] R. D. Jones, N. B. Sharman, R. W. Watson, and S. R. Muir, "A PCbased battery of tests for quantitative assessment of upper-limb sensory-motor function in brain disorders," Proc. 15th Int. Conf. IEEE Eng. Med. Biol. Soc., San Diego, 1993. 1414-1415.

[15] R. D. Jones, "Measurement of sensory-motor control performance capacities," in The Biomedical Engineering Handbook, vol. 2, J. D. Bronzino, Ed., 2 ed. Boca Raton, Florida: CRC Press, 2000, pp. 149:125.

[16] T. Higuchi, "Approach to an irregular time series on the basis of the fractal theory," Physica D: Nonlinear Phenomena, vol. 31, pp. 277 283, 1988.

[17] A. Delorme and S. Makeig, "EEGLAB: an open source toolbox for analysis of single-trial EEG dynamics including independent component analysis," Journal of Neuroscience Methods, vol. 134, pp. 9-21, 2004

[18] T.-P. Jung, S. Makeig, C. Humphries, T.-W. Lee, M. J. McKeown, V. Iragui, and T. J. Sejnowski, "Removing electroencephalographic artifacts by blind source separation," Psychophysiology, vol. 37, pp. 163-178, 2000.

[19] R. G. Lomax, An Introduction to Statistical Concepts for Education and Behavioral Sciences: Lawrence Erlbaum Associates, 2000.

[20] S. Makeig and T.-P. Jung, "Tonic, phasic, and transient EEG correlates of auditory awareness in drowsiness," Brain Res. Cogn. Brain Res., vol. 4, pp. 15-25, 1996. 